\title{
Subject areas for teaching GSS facilitation
}

\author{
R. B. Jarman \\ School of Information Systems, Curtin Business School \\ Curtin University of Technology, Perth Western Australia \\ E-Mail: bjarmanr@isis.curtin.edu.au
}

\section{Extended Abstract}

It has been proposed that 'strong facilitative and collaborative skills' will be required for the groupwork of the future, and that few institutions are offering training in these areas (Clawson \& Bostrom,1995, p189). If research suggests meetings are an increasing part of business life (Mosvick \& Nelson, 1987; Panko, 1992) and one in which substantial cost savings are available (Mosvick \& Nelson, 1987) education and training in these areas are important. But what should be taught to students? Research described below is identifying knowledge, skills and abilities required by novice meeting managers (students) to effectively lead meetings where a face-to-face Group Support Systems (GSS) is available.

\section{Research methodology}

In summary, there are five main stages to this research project:

(1) establishing a body of subject material to be taught to students, defining the material and developing the course;

(2) teaching the course, observing and discussing the students effectiveness in leading meetings, and with the input of the students, evaluating the material included in the course;

(3) refining the subject areas to be taught;

(4) seeking comments from experienced GSS facilitators and educators on the subject areas; and

(5) revise and finalise the subject areas and course in accordance with these suggestions.

At the time of writing the research is at Stage 3.

\section{Review of course development and evaluation}

A single semester course was developed based on meeting management, facilitation and GSS literature using Clawson's 16 facilitator roles (Clawson, 1992; Clawson \& Bostrom, 1995) as a starting point. The course contact was three hours per week over 14 teaching weeks. The structure was a 90 minute seminar followed by a 90 minute meeting. Areas covered included planning, designing and running meetings, communication skills, leadership, and problem solving and decision making processes. In the GSS area, results of the research were discussed including GSS effects such as anonymity and parallelism, and the factors which affect GSS meetings, and the effects on various 
processes and outcomes. There were eight students - five Asian, one African and two Australians. There were two females and six males. The multicultural background of the students is a factor of interest. Each student was part of a two-person facilitation team for two meetings. In these meetings, the students took turns in leading the meeting or being chauffeur. The GSS used in the meetings was GroupSystems V (for DOS).

The course has been evaluated both formally and informally. Formal evaluation was by questionnaire where students were be asked to evaluate the course based on what they learnt and how this equipped them to lead meetings. Informally, there was post-meeting evaluation and discussion. These evaluations, combined with the observations of the researcher, provide the basis for assessing how effectively the course achieved its objectives.

\section{Outcomes}

In summary, the students demonstrated they had acquired a variety of the facilitation skills but were not confident in actually administering the technology. In particular, the students were good at preparing and running the meetings, and ensuring there was an equality of participation, and that decisions were consensus decisions. The following points can be drawn from this phase of the research:

- the experience of running meetings is essential;

- in teaching students both facilitation skills and how to manage the tools careful consideration needs to be given as to how to achieve both within a single semester course;

- in brainstorming exercises some kind of arbitrary limit can be set to overcome information overload, and to ensure other aspects of meetings are also covered;

- teaching listening skills, and stressing the importance of the clarification of meanings is important to the idea organisation process;

- the importance of keeping to time versus the effects of rushing the group need to be highlighted;

- it may be beneficial to ensure the students are instructed in a number of specific process techniques rather than leaving it to their own reading;

- the stages of problem solving need to be emphasised so that problems are properly defined and criteria for evaluating solutions are developed before beginning the development of solutions;

- specific material on using 'questions' needs to be included;

- further methods for developing self awareness and self expression in a large group of students need to be examined, and included where appropriate;

- some students may require instruction on how to present information to a group;

- dealing with conflict appears a difficult area for teaching because of the experiential nature;

- inclusion of case studies where negotiation is required and with roles having conflicting interests is useful in highlighting where GSS may not be useful;

- subject material on GSS research does not appear to be relevant to facilitating;

- the opportunity to observe a variety of facilitator's would be beneficial;

- western facilitation, conflict management and feedback techniques may not be appropriate in Asian cultures

\section{Next phase}

At the time of writing, the course is being reviewed. A list of subject areas are being developed.

When complete it will be sent to experienced GSS facilitators and educators for rating as to importance and comments. On return of the advice from more experienced facilitator's the subject areas will be finalised. 